\title{
Automatic Prediction of Recurrence of Major Cardiovascular Events: A Text Mining Study Using Chest X-Ray Reports
}

\author{
Ayoub Bagheri ${ }^{10},{ }^{1}$ T. Katrien J. Groenhof, ${ }^{2}$ Folkert W. Asselbergs, ${ }^{3,4,5}$ Saskia Haitjema, ${ }^{6}$ \\ Michiel L. Bots, ${ }^{2}$ Wouter B. Veldhuis, ${ }^{7}$ Pim A. de Jong, ${ }^{7}$ and Daniel L. Oberski ${ }^{1,2}$ \\ ${ }^{1}$ Department of Methodology and Statistics, Faculty of Social Sciences, Utrecht University, Utrecht, Netherlands \\ ${ }^{2} J u l i u s$ Center for Health Sciences and Primary Care, University Medical Center Utrecht, Utrecht, Netherlands \\ ${ }^{3}$ Department of Cardiology, Division Heart and Lungs, University Medical Center Utrecht, Utrecht, Netherlands \\ ${ }^{4}$ Institute of Cardiovascular Science, Faculty of Population Health Sciences, University College London, London, UK \\ ${ }^{5}$ Health Data Research UK, Institute of Health Informatics, University College London, London, UK \\ ${ }^{6}$ Central Diagnostic Laboratory, University Medical Center Utrecht, Utrecht, Netherlands \\ ${ }^{7}$ Department of Radiology, Division of Imaging and Oncology, University Medical Center Utrecht, Utrecht, Netherlands
}

Correspondence should be addressed to Ayoub Bagheri; a.bagheri@uu.nl

Received 14 October 2020; Revised 29 May 2021; Accepted 29 June 2021; Published 9 July 2021

Academic Editor: Aiping Liu

Copyright $(92021$ Ayoub Bagheri et al. This is an open access article distributed under the Creative Commons Attribution License, which permits unrestricted use, distribution, and reproduction in any medium, provided the original work is properly cited.

Background and Objective. Electronic health records (EHRs) contain free-text information on symptoms, diagnosis, treatment, and prognosis of diseases. However, this potential goldmine of health information cannot be easily accessed and used unless proper text mining techniques are applied. The aim of this project was to develop and evaluate a text mining pipeline in a multimodal learning architecture to demonstrate the value of medical text classification in chest radiograph reports for cardiovascular risk prediction. We sought to assess the integration of various text representation approaches and clinical structured data with state-of-the-art deep learning methods in the process of medical text mining. Methods. We used EHR data of patients included in the Second Manifestations of ARTerial disease (SMART) study. We propose a deep learning-based multimodal architecture for our text mining pipeline that integrates neural text representation with preprocessed clinical predictors for the prediction of recurrence of major cardiovascular events in cardiovascular patients. Text preprocessing, including cleaning and stemming, was first applied to filter out the unwanted texts from X-ray radiology reports. Thereafter, text representation methods were used to numerically represent unstructured radiology reports with vectors. Subsequently, these text representation methods were added to prediction models to assess their clinical relevance. In this step, we applied logistic regression, support vector machine (SVM), multilayer perceptron neural network, convolutional neural network, long short-term memory (LSTM), and bidirectional LSTM deep neural network (BiLSTM). Results. We performed various experiments to evaluate the added value of the text in the prediction of major cardiovascular events. The two main scenarios were the integration of radiology reports (1) with classical clinical predictors and (2) with only age and sex in the case of unavailable clinical predictors. In total, data of 5603 patients were used with 5-fold cross-validation to train the models. In the first scenario, the multimodal BiLSTM (MI-BiLSTM) model achieved an area under the curve (AUC) of $84.7 \%$, misclassification rate of $14.3 \%$, and F1 score of $83.8 \%$. In this scenario, the SVM model, trained on clinical variables and bag-of-words representation, achieved the lowest misclassification rate of $12.2 \%$. In the case of unavailable clinical predictors, the MI-BiLSTM model trained on radiology reports and demographic (age and sex) variables reached an AUC, F1 score, and misclassification rate of $74.5 \%, 70.8 \%$, and $20.4 \%$, respectively. Conclusions. Using the case study of routine care chest X-ray radiology reports, we demonstrated the clinical relevance of integrating text features and classical predictors in our text mining pipeline for cardiovascular risk prediction. The MI-BiLSTM model with word embedding representation appeared to have a desirable performance when trained on text data integrated with the clinical variables from the SMART study. Our results mined from chest X-ray reports showed that models using text data in addition to laboratory values outperform those using only known clinical predictors. 


\section{Introduction}

Electronic health records (EHRs) data have become increasingly available to researchers as more hospitals, clinics, and practices have adopted data digitization. EHRs store data in different modalities, such as structured data (e.g., demographic values, laboratory results, and medications) and unstructured texts (e.g., referral letters, clinical notes, discharge summaries, and radiology reports). This digitization creates an opportunity to mine the health records to increase the quality of care and clinical outcomes. Yet, clinicians have limited time to process all the available data and detect patterns across similar medical records. Deep learning and machine learning, on the other hand, are suitable for discovering useful patterns from a vast amount of data.

Unstructured texts contained within the EHRs are recognized as a rich but not easily accessible and usable source of medical information [1-6]. Recent studies have attempted to derive information from unstructured medical texts to classify disease codes [7-10], detect patient's disease history [11, 12], and predict hospital readmission or clinical outcomes [13-15]. $\mathrm{X}$-ray radiology reports are an example of such unstructured data describing radiologist's observations on patient's medical conditions associated with medical images. The majority of previous decision support systems for radiology reports are developed using rule-based approaches applied on unstructured and semistructured texts [16-19]. However, these methods are often impractical because they do not generalize to new data and often are not applicable for big data analysis [20].

Recent studies have shown promising results using freetext radiology reports and deep learning models to predict clinical outcomes [17, 21-25]. Convolutional neural networks (CNNs) and recurrent neural networks (RNNs) are two common deep learning techniques that have been effective in text mining and natural language processing (NLP), as well as EHR applications [7, 15, 21, 26, 27]. Deep learning-based modelling of radiology reports has been proposed to supersede the simple grammatical patterns and hand-crafted regular expressions of the traditional clinical rule-based software, such as PEFinder [28], MedLEE [29, 30], and CTakes [31]. While these neural networks models gained tremendous momentum in knowledge discovery from EHR texts, there are very seldom studies that used both free-texts and structured information in EHRs for clinical prediction and classification [32-35].

In this paper, we leveraged structured features in EHR data to combine with free-text radiology reports to uncover patterns to improve cardiovascular risk prediction. Free-text within EHRs might contain additional information for clinical prediction modelling, either as an added variable to improve prediction performance compared to current models or as an auxiliary variable to increase the flexibility of prediction in the case of inaccessible clinical data.

The contributions of this study are twofold. The first contribution is to develop and evaluate a text mining pipeline for capturing additional information from text. The second is the use of chest X-ray reports from routine care as free-text in combination with the laboratory values, collected in the Second Manifestations of ARTerial disease (SMART) study [36], in a multimodal architecture to predict the recurrence of cardiovascular events in cardiovascular patients.

\section{Materials and Methods}

In this section, we describe the case study, data ethics and privacy, and the details of our proposed text mining pipeline.

\subsection{Case Study}

2.1.1. Patient Population. The patients included in this study were originally included in the SMART study. The design of the SMART study is published elsewhere [36]. In short, the SMART study is an ongoing single-center prospective cohort study designed to establish the presence of additional arterial disease and risk factors for atherosclerosis in patients with vascular disease or a vascular risk factor. Patients visiting the University Medical Center (UMC) Utrecht for evaluation of any atherosclerotic cardiovascular condition are eligible for inclusion in SMART. The inclusion criteria are presenting with an atherosclerotic cardiovascular condition and age $>18$ years. Exclusion criteria are life expectancy $<3$ months, unstable vascular disease, and insufficient fluency in the Dutch language. A total of 5603 SMART patients were included in this analysis. The characteristics of the patients are listed in Table 1.

2.1.2. Clinical Variables. Variables that are predictors in the SMART study [36] (age; sex; smoker; systolic blood pressure; diabetes; HDL cholesterol; total cholesterol; renal function according to the MDRD formula; history of cardiovascular disease stratified for stroke, peripheral artery disease, abdominal aortic aneurysm, and coronary heart disease; and years since diagnosis of first cardiovascular disease) were used for prediction modelling for all patients.

2.1.3. Chest $X$-Ray Reports. Free-text reports from chest X-rays that were taken in SMART patients, which were made in routine care, were extracted from their EHR and included in this analysis.

2.1.4. Ethics and Privacy. Informed consent was obtained through established procedures. The SMART study was approved by the Medical Ethical Committee of the UMC Utrecht. All data are handled according to local data protection guidelines and privacy regulations.

2.2. Text Mining Pipeline. Figure 1 illustrates the text mining pipeline for the prediction task. The goal is to forecast the major cardiovascular events (MACE) during follow-up as the outcome prior to clinical variables and chest X-ray reports.

2.2.1. Preprocessing. Clinical variables were preprocessed by missing value imputation and a normalization step. Missingness of data was solved using the MICE package [37] with one imputation for each missing value. As an additional 
TABLE 1: Characteristics of the patients.

\begin{tabular}{|c|c|}
\hline Characteristics & Total $n=5603$ \\
\hline Age, years, mean (SD) & $56.2(12.5)$ \\
\hline Female sex, $n(\%)$ & $1926(34.4)$ \\
\hline Current smoker, $n(\%)$ & $1549(27.6)$ \\
\hline \multicolumn{2}{|l|}{ History of CVD } \\
\hline $\mathrm{CHD}, n(\%)$ & $2166(38.7)$ \\
\hline Stroke, $n(\%)$ & $1076(19.2)$ \\
\hline $\mathrm{PAD}, n(\%)$ & $631(11.3)$ \\
\hline $\mathrm{AAA}, n(\%)$ & $306(5.5)$ \\
\hline $\begin{array}{l}\text { Years since first diagnosis of CVD, median } \\
\text { (IQR) }\end{array}$ & $0(0-4)$ \\
\hline \multicolumn{2}{|l|}{ Risk factors for CVD } \\
\hline Diabetes mellitus, $n(\%)$ & $1047(18.7)$ \\
\hline Hypertension, $n(\%)$ & $2353(42.0)$ \\
\hline Dyslipidemia, $n(\%)$ & $432(7.7)$ \\
\hline BMI, kg/m² (mean (SD)) & $26.8(4.3)$ \\
\hline SBP, mmHg (mean (SD)) & $140(21)$ \\
\hline $\mathrm{DBP}, \mathrm{mmHg}($ mean $(\mathrm{SD}))$ & $83(13)$ \\
\hline Total cholesterol, mmol/L (mean (SD)) & $5.14(1.38)$ \\
\hline LDL-cholesterol, mmol/L (mean (SD)) & $3.1(1.16)$ \\
\hline HDL-cholesterol, mmol/L (mean (SD) & $1.27(0.38)$ \\
\hline Triglycerides, mmol/L (median (IQR)) & $1.7(1.2-2.5)$ \\
\hline MDRD, $\mathrm{ml} / \mathrm{min} / 1.73 \mathrm{~m}^{2}$ (median (IQR)) & $80(68-91)$ \\
\hline $\mathrm{HbAlc}, \mathrm{mmol} / \mathrm{mol}$ (median (IQR)) & $5.7(5.4-6.1)$ \\
\hline Glucose, mmol/L (median (IQR)) & $5.7(2.6-6.4)$ \\
\hline Hemoglobin, mmol/L (mean (SD)) & $6.0(2.04)$ \\
\hline Creatinine, $\mu \mathrm{mol} / \mathrm{L}$ (median (IQR)) & $84(73-97)$ \\
\hline CRP, mg/L (median (IQR)) & $\begin{array}{c}1.95 \\
(0.90-4.20)\end{array}$ \\
\hline TSH, mU/l (mean (SD)) & $0.9(0.09)$ \\
\hline MACE during follow-up, $n(\%)$ & $1385(24.7)$ \\
\hline
\end{tabular}

CVD: cardiovascular disease; CHD: coronary heart disease; PAD: peripheral arterial disease; AAA: abdominal aortic aneurysm; BMI: body mass index; SBP: systolic blood pressure; DBP: diastolic blood pressure; LDL: low-density lipoprotein; HDL: high-density lipoprotein; MDRD: modification of diet in renal disease; HbAlc: hemoglobin; Alc CRP: C-reactive protein; TSH: thyroid-stimulating hormone; MACE: major cardiovascular events.

normalization step, the clinical variables were rescaled to homogenize their levels of variance.

In preprocessing the radiology reports, the following steps were performed to improve the quality of text data for the subsequent steps: (1) all characters were transformed into lowercase; (2) we removed numbers and some meaningless punctuation marks, such as semicolons and colons; and (3) stop words were then removed. Dutch stop words used in this study are shown in Table 2. (4) We then applied Porter's stemming algorithm $[38,39]$ to texts. Figure 2 shows the 20 most frequent words before and after the preprocessing step for the X-ray radiology report in the SMART study. "Klinisch (clinical)" and "xthorax (chest X-ray)" appeared in all reports as they denote the indication of the test and type of X-ray; therefore, we removed them as noninformative stop words. Other words were merged into their stem words.

2.2.2. Representation and Feature Extraction. Text representation includes dimensions in which text is represented in a vector space model. We explored three text representation techniques used in the text mining pipeline: (i) Bag-of-words (BOW)

(ii) Clustering-based representation

(iii) Word embedding

We used three different techniques: an interpretable method, a method with a low-dimensional output, and a less interpretable and more semantic-based technique to be able to assess their differences in performance in mining additional information for clinical prediction modelling.

(i) Bag-of-Words. The BOW representation is the most commonly used representation for text mining applications [11]. Words in the reports were converted into a sparse multidimensional representation, which was leveraged for further classification and clustering purposes. Representation of text includes frequencies of words per patient's text report. This is a method that is relatively easy to understand and interpret for clinicians.

(ii) Clustering-Based Representation. We applied latent Dirichlet allocation (LDA) [40] to further cluster the BOW representation of patient radiology reports. LDA is a topic modelling approach that groups a collection of documents to obtain the probabilities of the distributions of document-topic and topic-word in the data set. This method has the advantage of using an interpretable lower-dimensional representation of text and the disadvantage of lacking the capacity of methods that use all features of unstructured medical notes. We ran the experiments fitting the LDA topic model with Gibbs sampling [11, 40] using 10 topics. Figure 3 shows two topics of the output of LDA applied to the X-ray radiology reports in the SMART study. Potential clinical scenarios that fit these topics are (a) possible cardiac decompensation and (b) possible pneumonia.

(iii) Word Embedding. Neural network-based word embedding incorporates not only the contexts of a word but also the semantic relation with other words $[1,41,42]$. We used a window of five words for the context words that were used in this representation technique. Subsequently, word vectors were aggregated for each patient report.

2.2.3. Classification Algorithms. In the text mining pipeline, independent variables are the clinical variables and features extracted from radiology reports, though these features differ per text representation approach. MACE as defined by the SMART study was used as an outcome variable. We made a total of six different algorithms to be able to study both baseline and the state-of-the-art machine learning methods and their additional value for clinical risk prediction.

(i) Baseline Models. Using traditional machine learning classifiers, we applied an LR model and a support vector machine (SVM) algorithm to data from the SMART case study. If the interpretation of a model is of primary interest, LR parameters can easily be interpreted in terms of the log odds. SVM on the other hand is a supervised learning technique that produces nonlinear boundaries by constructing a linear boundary in a large, transformed version of 


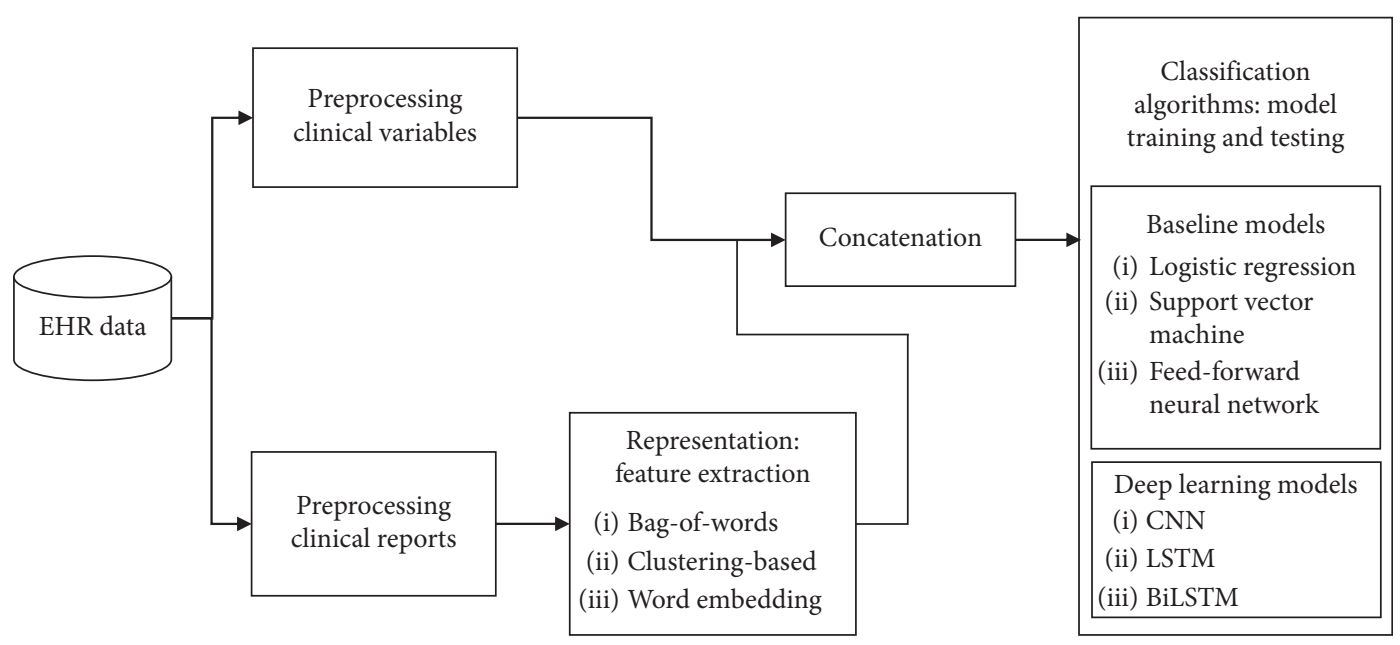

FIGURE 1: Methodology text mining pipeline overview.

TABle 2: Dutch stop words used in this case study.

\begin{tabular}{|c|c|c|c|c|c|c|}
\hline de & informatie & je & $\mathrm{al}$ & na & worden & tegen \\
\hline en & eerdere & mij & waren & reeds & zelf & gegevens \\
\hline van & klinisch & uit & doen & wil & ons & klinische \\
\hline $\mathrm{ik}$ & er & der & toen & kon & kunnen & tot \\
\hline te & maar & daar & moet & uw & ook & omdat \\
\hline dat & om & haar & ben & iemand & bij & ge \\
\hline die & hem & naar & kan & geweest & zich & $\mathrm{nu}$ \\
\hline in & dan & heb & hun & andere & gegevens & had \\
\hline aan & zou & hoe & dus & klinisch & voor & als \\
\hline een & of & heeft & onder & informatie & hier & thorax \\
\hline hij & wat & hebben & ja & gegeven & men & $\mathrm{u}$ \\
\hline het & mijn & deze & eens & xthorax & zijn & doch \\
\hline is & dit & want & wie & conclusie & met & me \\
\hline was & zo & nog & werd & onderzoek & ze & zij \\
\hline op & door & $\mathrm{zal}$ & altijd & opname & wordt & eerder \\
\hline over & ter & $\mathrm{x} / \mathrm{x}$ & & & & \\
\hline
\end{tabular}

the feature space, and it scales relatively well to high dimensional data, such as unstructured texts [43].

(ii) Deep Learning Models. We studied using the state-of-theart deep learning methods: a CNN, a long short-term memory (LSTM) RNN, and a bidirectional LSTM (BiLSTM) $[7,15,21,26]$. We also employed a feed-forward multilayer perceptron neural network for the case of no text presented to the model. However, multilayer perceptron is not well adapted to textual data $[1,8,26]$. This is because it is defined for vectors as input data; hence, to apply it to texts, we must transform the texts into vectors. CNN, LSTM, and BiLSTM are deep learning architectures that have removed the manual extraction of features from text data.

2.2.4. Multimodal Neural Network. Figure 4 illustrates the proposed deep learning-based architecture for the text mining pipeline. In this architecture, we propose a multimodal learning model using a BiLSTM deep neural network.

The multimodal neural network architecture consists of an embedding layer, a BiLSTM layer, a dropout, a concatenation layer, and dense layers.
2.2.5. Embedding Layer. To extract the semantic information of radiology reports, each text is firstly represented as a sequence of word embeddings. Word embedding is an improvement over the bag-of-words models where large sparse vectors were used to represent each word. On the contrary, in an embedding, words are represented by dense vectors where a vector represents the projection of the word into a continuous vector space $[41,42]$. Denote $s$ as an X-ray report with $m$ words and each word is mapping to a vector; then, we have

$$
s=\left[\vec{e}_{1}, \vec{e}_{2}, \ldots, \vec{e}_{m}\right]
$$

where vector $\vec{e}_{i}$ represents the vector of $i$-th word with a dimension of $d$. The vectors of word embeddings are concatenated together to maintain the order of words in a patient report.

2.2.6. Bidirectional-LSTM Layer. After the embedding layer, the sequence of word vectors is fed into a bidirectional LSTM layer to achieve another representation of radiology reports. Interest in incorporating a BiLSTM layer into the architecture of our model arises from their ability to learn long-term dependencies and contextual features from both past and future states [44]. The BiLSTM layer calculates two parallel LSTM 


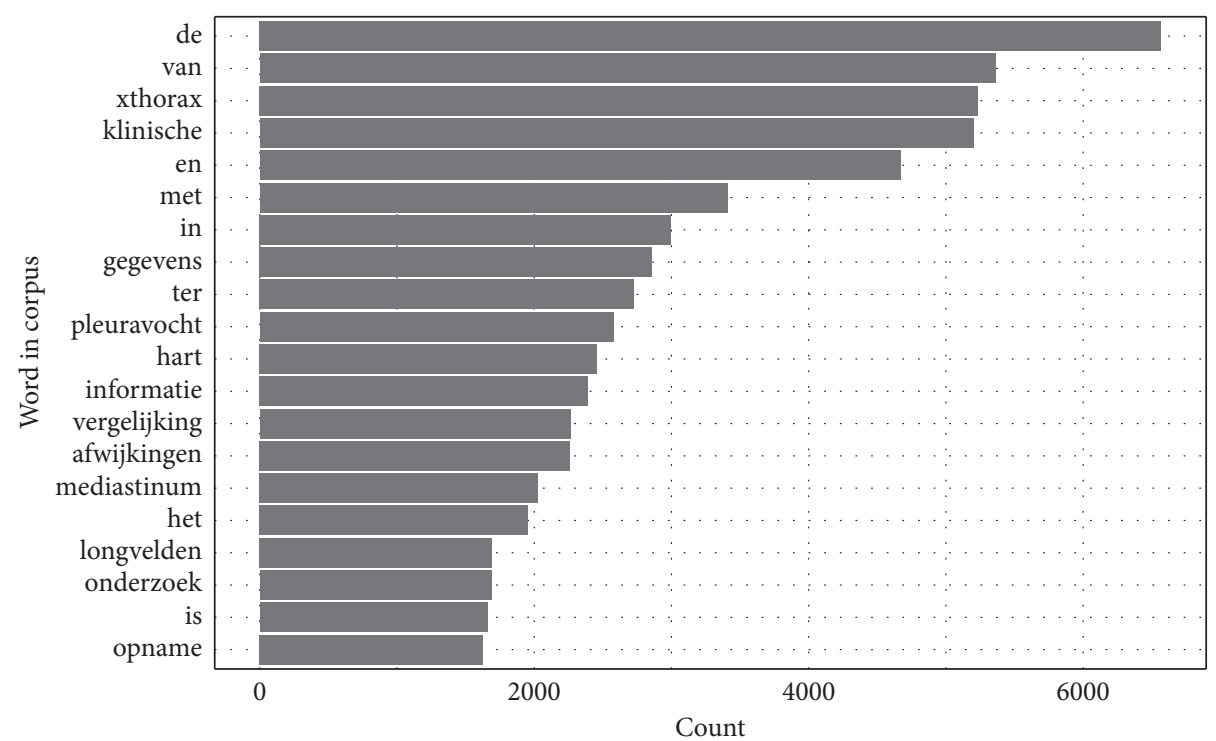

(a)

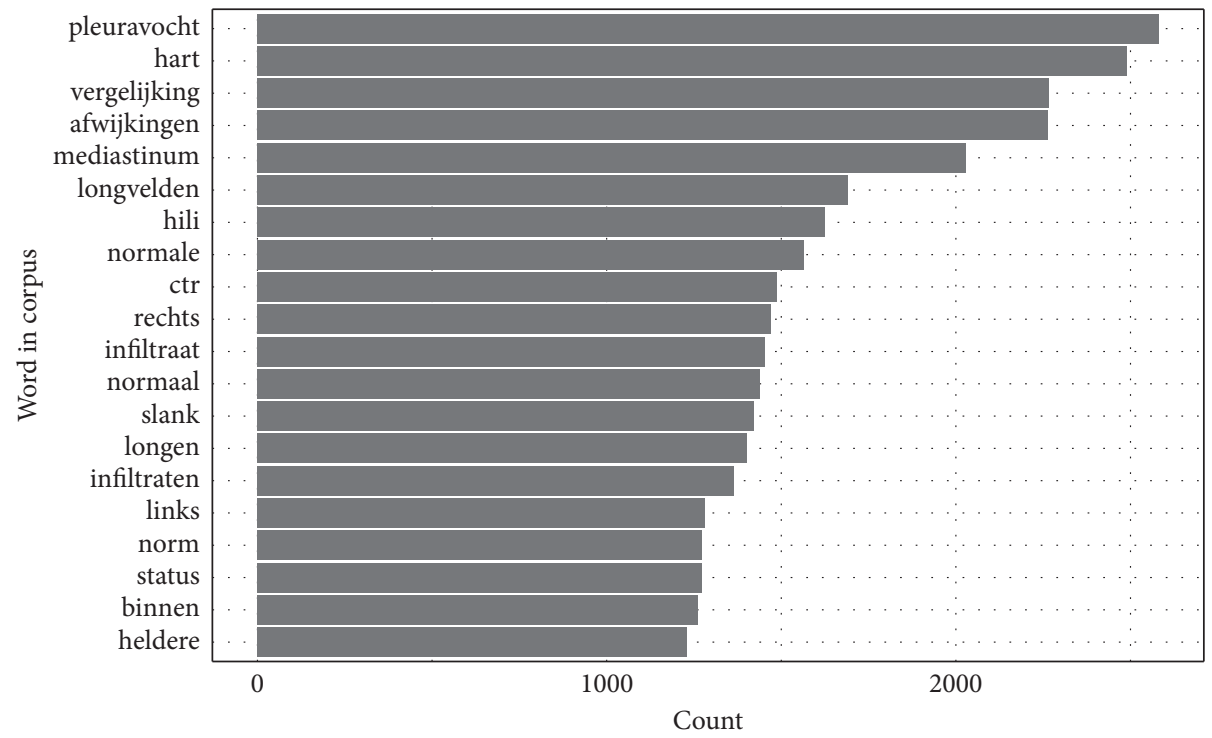

(b)

FIgURE 2: Most frequent words in the X-ray radiology reports in the SMART study. (a) Initial top frequent words. (b) Top frequent words after preprocessing.

layers, a forward hidden layer, and a backward hidden layer, to generate an output sequence $y$ as illustrated:

$$
\begin{aligned}
h_{f_{t}} & =\sigma\left(W_{x h_{f}} x_{t}+W_{h_{f} h_{f}} h_{f_{t-1}}+b_{h_{f}}\right), \\
h_{b_{t}} & =\sigma\left(W_{x h_{b}} x_{t}+W_{h_{b} h_{b}} h_{b_{t-1}}+b_{h_{b}}\right), \\
y_{t} & =W_{h_{f} y} h_{f_{t}}+W_{h_{b} y} h_{b_{t}}+b_{y},
\end{aligned}
$$

where $\sigma$ is the sigmoid activation function; $x t$ is a $d$-dimensional input vector at time step $t ; W$ are the weight matrices; $b$ are bias vectors; and $h_{f}$ and $h_{b}$ are the output of the LSTM forward and backward layers, respectively.

The multimodal BiLSTM integrates the neural text representation with clinical predictors and feeds them into a fully connected neural network. We used a BiLSTM network to connect both previous and future information to the present information in text reports. This was made possible by having two propagating networks in opposite directions: one network running from the beginning of the text to the end and the other in the opposite direction. These forward and backward networks memorize information about the report from both directions. Thus, the context window around each word consists of information both prior to and after the current word. In this way, BiLSTM can model the entire sequence of words in a radiology report to capture dependencies between the feature space and the relationship with the outcome variable.

2.2.7. Other Deep Neural Networks. When applying a CNN model to our architecture, we used a convolution layer with a 


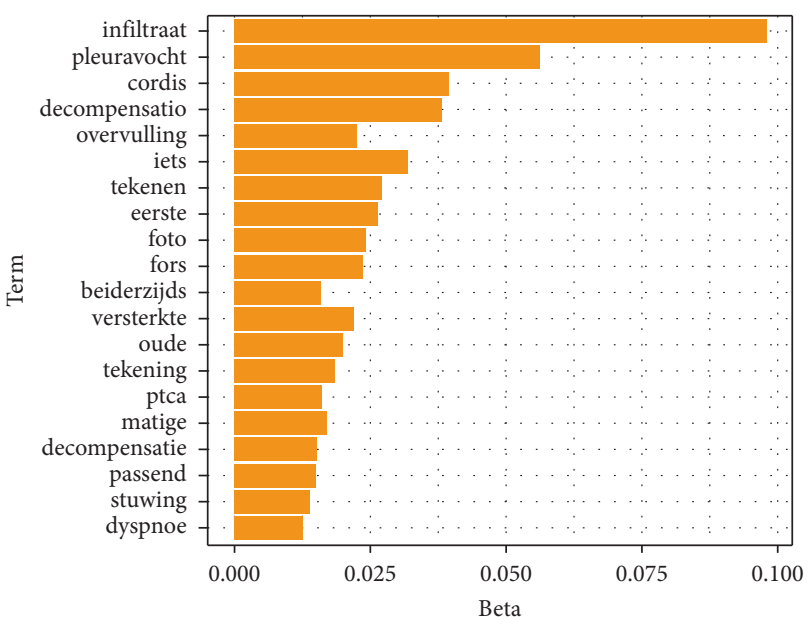

(a)

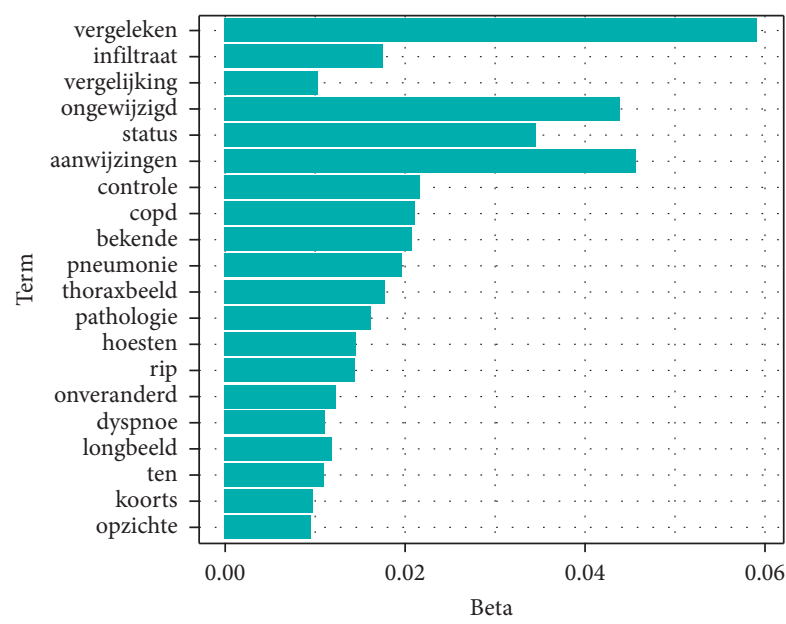

(b)

FIgURE 3: LDA clustering. The $y$-axis shows the top words in the selected cluster (topic). The $x$-axis shows the probability of the word in the topic. (a) Possible cardiac decompensation. (b) Possible pneumonia.

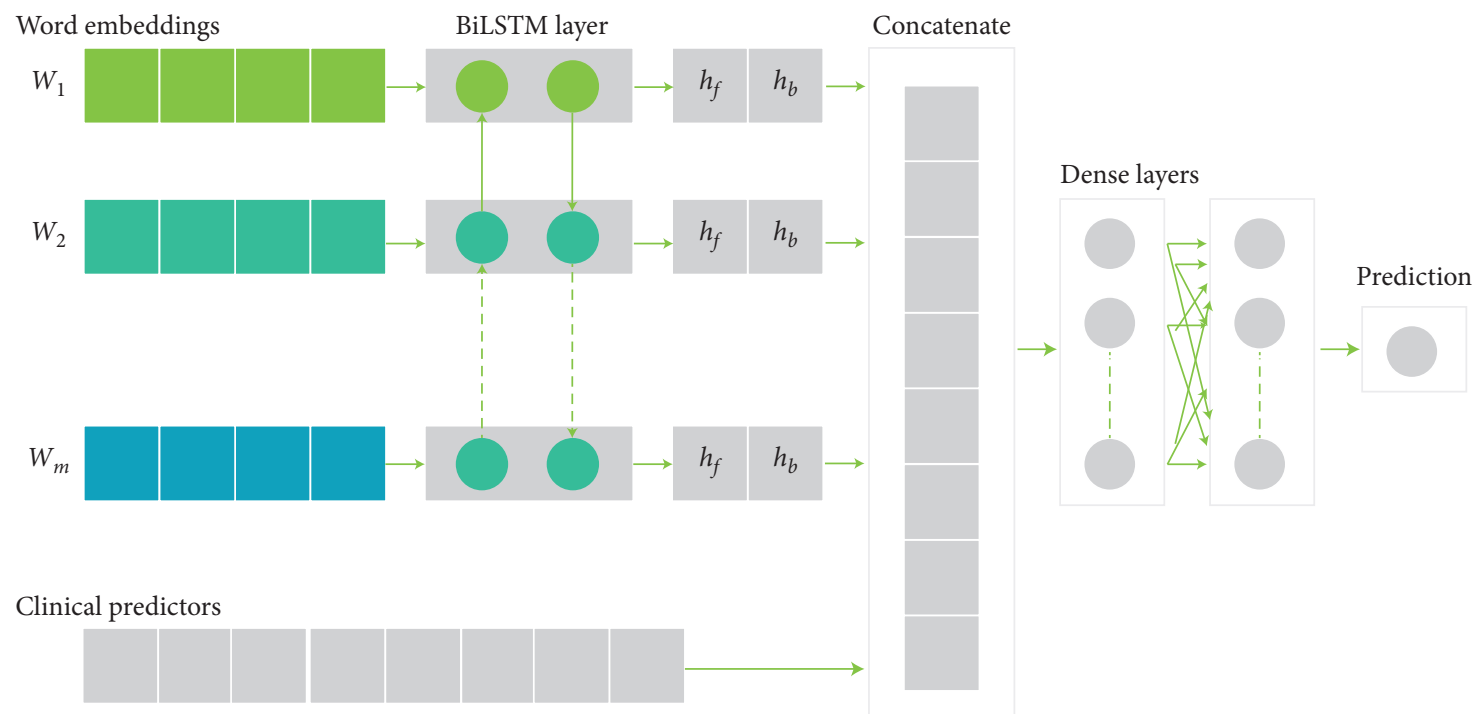

Figure 4: Proposed multimodal learning architecture with a deep learning model.

max pooling layer instead of the BiLSTM layer in the architecture in Figure 4. For employing an LSTM model, only the left to the right direction in the text is monitored inside the hidden RNN layer.

2.3. Evaluation Measures. To evaluate the classification performance of our text mining pipeline, we used five available metrics: area under the curve (AUC), misclassification rate, precision (positive predictive value), recall (sensitivity), and F1 score. AUC is the area under the receiver operating characteristic curve, which is created by plotting the true positive rate against the false positive rate. Misclassification rate is the proportion of incorrectly classified instances made by a model. Precision is the fraction of relevant instances among the retrieved instances, while recall is the fraction of relevant instances that have been retrieved over the total amount of relevant instances. The F1 score can be interpreted as a weighted average of precision and recall. The relative contributions of precision and recall to the F1 score are equal. The formulae of precision, recall, and the F1 score are defined in the following:

$$
\begin{aligned}
\text { precision } & =\frac{\text { true positive }}{\text { true positive }+ \text { false positive }}, \\
\text { recall } & =\frac{\text { true positive }}{\text { true positive }+ \text { false negative }}, \\
\mathrm{F} 1 \text { score } & =\frac{2 * \text { precision } * \text { recall }}{\text { precision }+ \text { recall }} .
\end{aligned}
$$




\section{Results}

Our pipeline was implemented in Python and $R$ using various text mining, NLP, and machine learning packages. The multimodal learning architecture was implemented on Keras with a TensorFlow backend (https://keras.io). The source code is publicly available at GitHub (https://github. com/bagheria/CardioRisk-TextMining). We performed 5fold cross-validation for all experimental analyses. We used the hyperparameter setting as shown in Table 3. These hyperparameters were tuned based on the validation set. We used the embeddings with a vector size equal to 500 and a window size equal to 5 . In addition, we set the number of filters in the CNN to 128 and the filter size to 5. The hidden dense layers contained 64 units and used the ReLU activation function, and the output layer used a sigmoid activation function. We set the same number of hidden units in the LSTM layers at 100. Both dropout and recurrent dropout were added at 0.2 to avoid overfitting [45]. We set the batch size and number of epochs to 64 and 20, respectively.

To assess the added value of text for the prediction of MACE, we compared various scenarios of clinical variables and text reports in the proposed text mining pipeline:

(1) Prediction using only radiology reports (models starting with $\mathrm{T}$ )

(2) Prediction using only clinical variables (models starting with $\mathrm{V}$ )

(3) Prediction using the integration of clinical variables and radiology reports (models starting with VB, VC, and MI)

(4) Prediction using only sex and age variables (models starting with D)

(5) Prediction using the integration of sex and age variables and radiology reports (models starting with $\mathrm{DB}, \mathrm{DC}$, and $\mathrm{D}-\mathrm{MI})$

Table 4 lists the experimental results for AUC and the misclassification rate for the first three scenarios. In these experiments, we evaluated different models using only clinical variables, only radiology reports, and their integration.

V-LR, V-SVM, and V-NN are the models trained on only clinical variables. The features in these models included the SMART variables as independent variables and MACE during follow-up as the outcome in prediction models. T-SVM, T-LR, and T-BiLSTM are the models with only text reports as their predictors. T-SVM was trained on the BOW representation of the reports. T-LR used the clustering-based representation. In this scenario, we reported each model's best result among representation methods. T-SVM achieved the highest performance in this scenario with an AUC of $62.5 \%$ and a misclassification rate of $18.6 \%$. VB and VC are the models trained on clinical variables combined with the BOW and clusteringbased representations, respectively. VC-SVM gained the lowest AUC of $65.5 \%$, while the VB-SVM model obtained the lowest misclassification rate of $12.2 \%$.

MI represents the models that used the proposed multimodal learning architecture with the neural word embedding representation. In this scenario, MI-BiLSTM,
TABLE 3: Hyperparameter setting.

\begin{tabular}{lc}
\hline Hyperparameter & Value \\
\hline Embedding size & 500 \\
Window size & 5 \\
\#filters & 128 \\
Filter size & 5 \\
\#hidden units & 64 \\
Hidden activation function & ReLU \\
Output activation function & Sigmoid \\
\#LSTM units & 100 \\
Dropout & 0.2 \\
Recurrent dropout & 0.2 \\
Batch size & 64 \\
\#epochs & 20 \\
\hline
\end{tabular}

TABle 4: Performance comparison of different experimental scenarios using AUC and misclassification rate.

\begin{tabular}{lcc}
\hline Classifier & AUC & Misclassification rate \\
\hline V-LR & 0.799 & 0.195 \\
V-SVM & 0.648 & 0.196 \\
V-NN & 0.651 & 0.201 \\
T-LR & 0.512 & 0.247 \\
T-SVM & 0.625 & 0.186 \\
T-BiLSTM & 0.570 & 0.300 \\
VB-LR & 0.808 & 0.193 \\
VB-SVM & 0.784 & 0.122 \\
VC-LR & 0.809 & 0.194 \\
VC-SVM & 0.655 & 0.197 \\
MI-LR & 0.811 & 0.203 \\
MI-SVM & 0.694 & 0.237 \\
MI-CNN & 0.730 & 0.214 \\
MI-LSTM & 0.794 & 0.176 \\
MI-BiLSTM & 0.847 & 0.143 \\
\hline
\end{tabular}

MI-LSTM, and MI-LR achieved promising results. MIBiLSTM obtained the highest AUC of $84.7 \%$ and the lowest misclassification rate of $14.3 \%$ in this case. MI-LR still has the second ranking AUC at $81.1 \%$.

Precision, recall, and F1 score evaluation measures are recommended for imbalanced data, where the AUC and misclassification rate may provide an optimistic view of the performance [46]. Figure 5 shows the performance of the models using precision, recall, and F1 score metrics. The deep learning models achieved better performance compared to other models in different scenarios. The MIBiLSTM model achieved the highest performance in terms of all evaluation measures. The F1 score was $83.8 \%$. MILSTM and MI-CNN obtained F1 score performances of $78.9 \%$ and $74.7 \%$, respectively. These results are evidence of the performance of text mining techniques with multimodal learning architecture in extracting knowledge from radiology reports and combining them with classical clinical predictors. It is notable that the multilayer perceptron neural network achieved promising results when trained only on clinical variables. This model obtained a precision of $75.1 \%$, recall of 79.4, and F1 score of $77.2 \%$. This shows the efficiency of the neural network model and the relatedness of the laboratory results in predicting cardiovascular risk. 


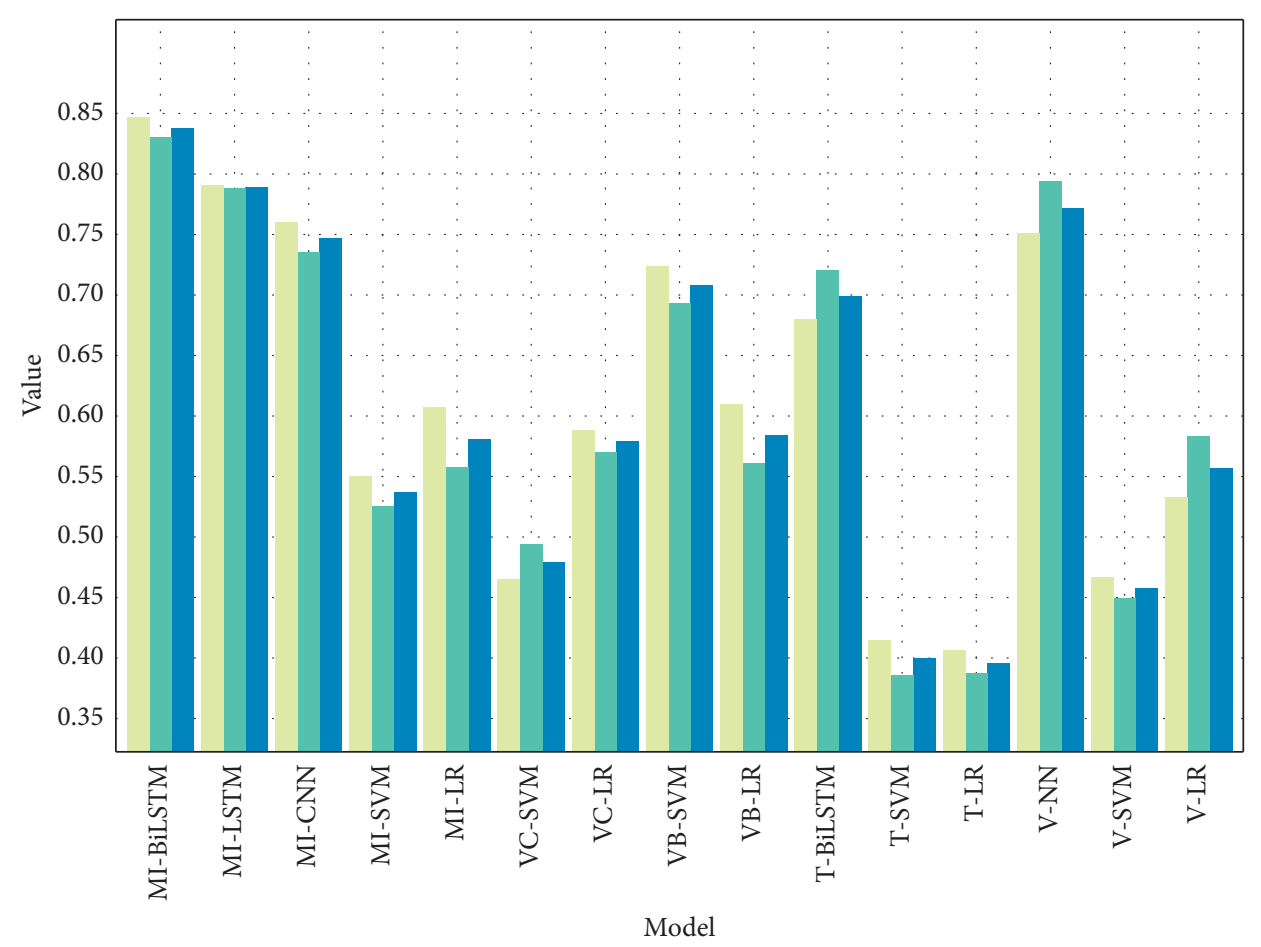

Metric

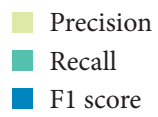

FIGURE 5: Comparison of precision, recall, and F1 score for experimental scenarios.

To assess the value of text as additional variables if clinical predictors are not available, we again compared the abovementioned scenarios but with only sex and age as clinical variables. Table 5 lists the results of this evaluation of the text mining pipeline. We named the models in this scenario D-models to show that they have been trained on demographic (age and sex) features.

The D-MI-BiLSTM model gained the highest AUC of $74.5 \%$. D-MI-BiLSTM was trained using the multimodal architecture, meaning that it used the neural word embedding representation and BiLSTM hidden layer output to concatenate the radiology reports with age and sex. DB-SVM gained the lowest misclassification rate of $16.3 \%$. This model was trained on the combination of the $\mathrm{BOW}$ representation and the age and sex variables.

In Figure 6, the results of precision, recall, and F1 score are compared for the scenarios when clinical predictors are not available. This setting also confirms that text miningbased models achieved better performance when predicting the MACE variable. The D-MI-BiLSTM, D-MI-LSTM, and D-MI-CNN models gained F1 scores of $70.8 \%, 67 \%$, and $64.3 \%$, respectively. The LR model with only age and sex only reached $44.2 \%, 49.4 \%$, and $46.66 \%$, respectively.

\section{Discussion}

This study aimed to develop and evaluate a text mining pipeline integrating clinical and text variables applied to
TABle 5: Performance comparison of different experimental scenarios using AUC and misclassification rate when clinical predictors are not available.

\begin{tabular}{lcc}
\hline Classifier & AUC & Misclassification rate \\
\hline D-LR $^{a}$ & 0.685 & 0.242 \\
D-SVM & 0.572 & 0.246 \\
D-NN & 0.567 & 0.214 \\
DB-LR $^{b}$ & 0.703 & 0.247 \\
DB-SVM $^{-}$ & 0.674 & 0.163 \\
DC-LR $^{c}$ & 0.705 & 0.239 \\
DC-SVM $^{d}$ & 0.534 & 0.247 \\
D-MI-LR & 0.708 & 0.235 \\
D-MI-SVM & 0.568 & 0.247 \\
D-MI-CNN & 0.667 & 0.228 \\
D-MI-LSTM & 0.708 & 0.209 \\
D-MI-BiLSTM & 0.745 & 0.204 \\
\hline
\end{tabular}

${ }^{a}$ LR trained on demographic variables. ${ }^{b}$ LR trained on demographic variables and BOW representation. ${ }^{c} \mathrm{LR}$ trained on demographic variables and clustering-based representation. ${ }^{d}$ Multimodal learning LR trained on demographic variables and word embeddings.

cardiovascular risk prediction. Our research (1) integrates EHR structured laboratory results and unstructured radiology text in a text mining pipeline to make an accurate decision for classifying cardiovascular events; (2) uses routine care data including X-ray reports in Dutch for cardiovascular risk prediction; and (3) incorporates free-text reports as auxiliary variables when classical predictors are not available. In our experiments for the SMART case study, 


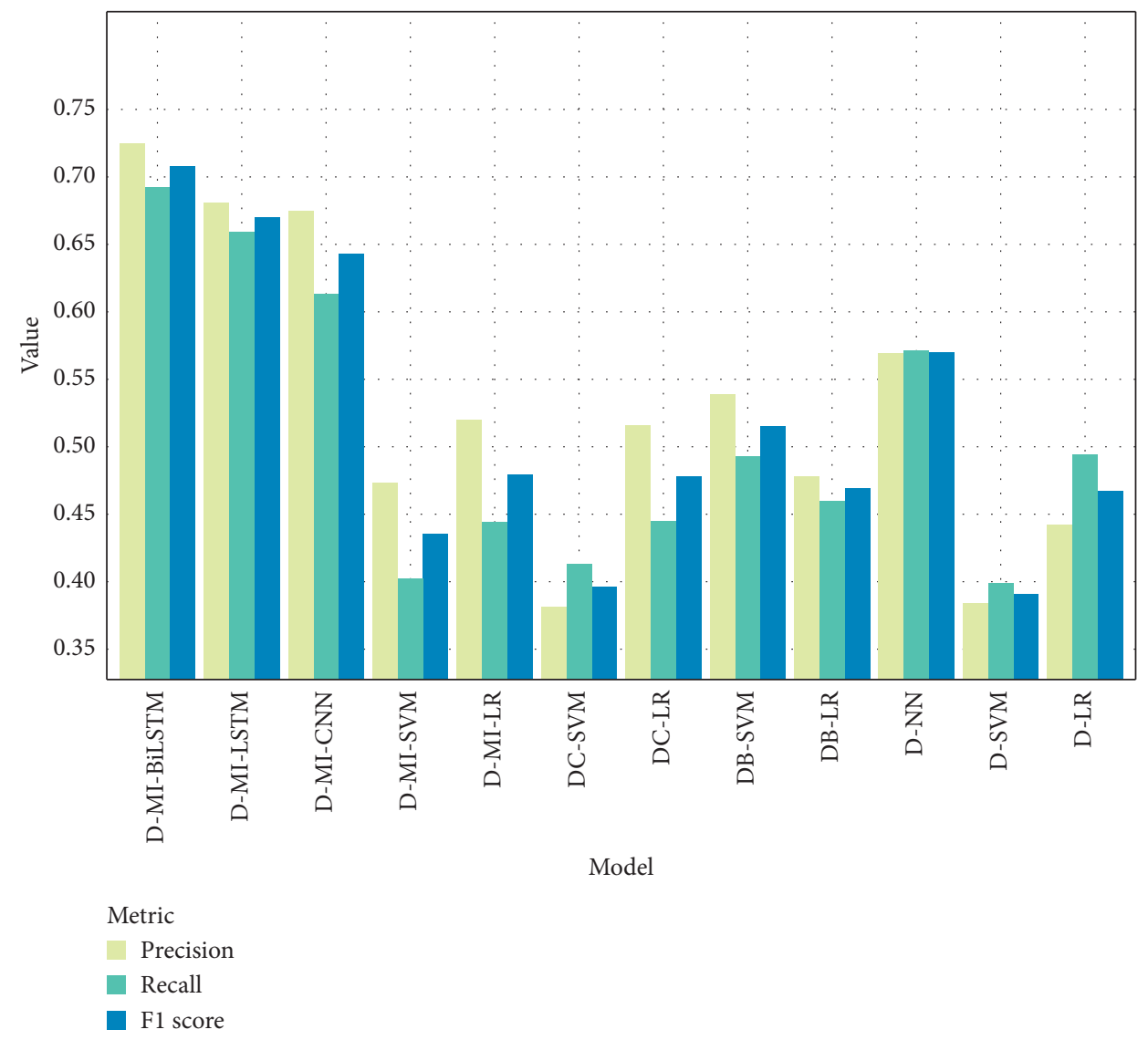

Figure 6: Comparison of precision, recall, and F1 score for experimental scenarios when clinical predictors are not available.

we found that neural text representation and prediction modelling significantly add to baseline models with classical clinical predictors to predict MACE. In the case of unavailable clinical predictors, the proposed MI-BiLSTM model with just age, sex, and word embedding attains a similar discriminative performance to that of the models trained on the classical SMART variables. Deep learning methods are increasingly being adopted in the medical field. For example, in radiology, deep learning has shown remarkable results in image analysis [47], and in intensive care, RNNs have been used to determine variables that are proxies for clinician decision-making [48]. The application of text mining and NLP in the predictive setting is not new; unlocking the full potential of EHR data is contingent on the development of text mining pipelines to automatically transform free-text into structured clinical data that can guide clinical decisions $[1,2,4,49]$. Yet, text as auxiliary variables to classical clinical variables has only been considered in a few studies [32-34, 50, 51]. One study [51] predicted several clinical interventions combining structured data and clinical notes. Each clinical narrative note was transformed to a 50-dimensional vector of topic proportions for each note using an LDA algorithm. This resulted in a lower-dimensional representation of text, losing the depth of information in unstructured text. Another study [33] extracted structured information from clinical notes using regular expression and a heuristic rule-based tool. Again, this is a method that uses a general framework for predicting the onset of diseases, combining both free-text medical notes and structured information. The mined text was then used to predict congestive heart failure, kidney failure, and stroke via deep learning models, achieving good performance in disease prediction. Lastly, one study [34] combined unstructured text, semistructured text, and structured data in machine learning models. Separate models were developed to handle data from different modalities to create an ensemble model that predicts diagnostic codes of the international classification of diseases (ICD-10). Hence, in this study, we combine all advantages of prior research by developing a machine learning-based modelling of radiological language, to integrate clinical variables and textual features, to supersede traditional algorithms using only clinical variables. In this paper, we explained how we used text preprocessing techniques and applied text representation methods to chest X-ray reports. These representations were then used as auxiliary variables to the clinical variables from the SMART study to predict MACE using six different classification techniques.

There are strengths and limitations to our case study. Because patients must have had an indication for a routine care chest X-ray, there was a selection in the case study. However, this does not in fact mean there is selection bias; it merely restricts the generalizability of the clinical prediction model to cardiovascular patients without an X-ray report 
available. Pragmatically, we hypothesize that using available information-including bodies of text, such as this chest $\mathrm{X}$-ray report-for predictions rather than a strict set of predictors will make predictions more flexible and more tailored to individuals. The use of advanced techniques, such as text mining, in clinical practice requires support for implementation. Implementation includes the application of the mining pipeline and integration in the care process using technologies, such as computerized decision support (CDSS). CDSS allows technical results from algorithms, such as text mining, to be translated to practical suggestions for clinical practice. To help clinicians interpret results that come from text mining, collaborations between technical text mining experts (biostatisticians, mathematicians, data scientists, and software engineers) and practical experts (clinicians) are needed to safeguard the technical quality and medical relevance. Future studies will focus on two points. First, our multimodal learning architecture will be validated for other similar scenarios, such as adverse event monitoring, hospital readmission, or disease classification, in which both EHR structured variables and free-text reports would contribute to the judgement of final outcomes. Secondly, we will expand our pipeline to a model to use the available clinical dictionaries with machine learning and deep learning models. The publicly available source code of our model (https://github.com/bagheria/CardioRiskTextMining) can be used to evaluate performance on potentially clinically relevant classification tasks based on clinical notes and EHR variables.

\section{Conclusions}

Medical free-text potentially contains valuable information for clinical decision-making. Text mining methods are the key to the successful extraction of clinically important findings from these free-text reports. Medical text mining is a step-by-step process that requires tailoring to the aim of the project and the context of reports. Text mining potentially opens the door to valuable information captured in free-text medical data. We believe that such models are useful in reducing work overload for clinicians by providing the needed clinical decision support.

\section{Data Availability}

The dataset is not publicly available due to patient privacy restrictions.

\section{Conflicts of Interest}

The authors declare that they have no conflicts of interest.

\section{Acknowledgments}

This work received support from the EU/EFPIA Innovative Medicines Initiative 2 Joint Undertaking BigData@Heart (Grant no. 116074). Folkert Asselbergs was supported by UCL Hospitals NIHR Biomedical Research Centre. The Department of Radiology partly received research support from Philips Healthcare.

\section{References}

[1] S. Sheikhalishahi, R. Miotto, J. T. Dudley, A. Lavelli, F. Rinaldi, and V. Osmani, "Natural language processing of clinical notes on chronic diseases: systematic review," JMIR Medical Informatics, vol. 7, no. 2, Article ID e12239, 2019.

[2] I. Drozdov, D. Forbes, B. Szubert, M. Hall, C. Carlin, and D. J. Lowe, "Supervised and unsupervised language modelling in chest x-ray radiological reports," PLoS One, vol. 15, no. 3, Article ID e0229963, 2020.

[3] J. Zech, M. Pain, J. Titano et al., "natural language-based machine learning models for the annotation of clinical radiology reports," Radiology, vol. 287, no. 2, pp. 570-580, 2018.

[4] Z. Wang, A. D. Shah, A. R. Tate, S. Denaxas, J. Shawe-Taylor, and H. Hemingway, "Extracting diagnoses and investigation results from unstructured text in electronic health records by semi-supervised machine learning," PLoS One, vol. 7, no. 1, Article ID e30412, 2012.

[5] J. Pérez, A. Pérez, A. Casillas, and K. Gojenola, "Cardiology record multi-label classification using latent dirichlet allocation," Computer Methods and Programs in Biomedicine, vol. 164, pp. 111-119, 2018.

[6] A. Bagheri, Text mining in healthcare: bringing structure to electronic health records, Ph.D. thesis, Utrecht University, Utrecht, Netherlands, 2021.

[7] J. Du, Q. Chen, Y. Peng, Y. Xiang, C. Tao, and Z. Lu, "Ml-net: multi-label classification of biomedical texts with deep neural networks," Journal of the American Medical Informatics Association, vol. 26, no. 11, pp. 1279-1285, 2019.

[8] A. Bagheri, A. Sammani, P. G. Van der Heijden, F. W. Asselbergs, and D. L. Oberski, "Automatic icd-10 classification of diseases from Dutch discharge letters," in Proceedings of the 13th International Joint Conference on Biomedical Engineering Systems and Technologies, pp. 281289, Valletta, Malta, February 2020.

[9] A. Blanco, O. Perez-de-Viñaspre, A. Pérez, and A. Casillas, "Boosting icd multi-label classification of health records with contextual embeddings and label-granularity," Computer Methods and Programs in Biomedicine, vol. 188, Article ID 105264, 2020.

[10] J. Huang, C. Osorio, and L. W. Sy, "An empirical evaluation of deep learning for icd-9 code assignment using mimic-iii clinical notes," Computer Methods and Programs in Biomedicine, vol. 177, pp. 141-153, 2019.

[11] A. Bagheri, A. Sammani, P. Van Der Heijden, F. W. Asselbergs, and D. L. Oberski, "Etm: enrichment by topic modeling for automated clinical sentence classification to detect patients' disease history," Journal of Intelligent Information Systems, vol. 55, 2020.

[12] X. Wu, Y. Zhao, D. Radev, and A. Malhotra, "Identification of patients with carotid stenosis using natural language processing," European Radiology, vol. 30, pp. 1-9, 2020.

[13] B. Alex, C. Grover, R. Tobin, C. Sudlow, G. Mair, and W. Whiteley, "Text mining brain imaging reports," Journal of Biomedical Semantics, vol. 10, no. 1, pp. 23-34, 2019.

[14] K. Huang, J. Altosaar, and R. Ranganath, "Clinicalbert: modeling clinical notes and predicting hospital readmission," 2019, https://arxiv.org/abs/1904.05342.

[15] A. N. Jagannatha and H. Yu, "Bidirectional rnn for medical event detection in electronic health records," in Proceedings of the NAACL, HLT 2016 Association for Computational Linguistics Conference, p. 473, June 2016.

[16] T. Gong, C. L. Tan, T. Y. Leong et al., "Text mining in radiology reports," in Proceedigs of the 8th IEEE International 
Conference on Data Mining, pp. 815-820, IEEE, Pisa, Italy, December 2008.

[17] M. C. Chen, R. L. Ball, L. Yang et al., "Deep learning to classify radiology free-text reports," Radiology, vol. 286, no. 3, pp. 845-852, 2018.

[18] R. K. Taira, S. G. Soderland, and R. M. Jakobovits, "Automatic structuring of radiology free-text reports," Radiographics, vol. 21, no. 1, pp. 237-245, 2001.

[19] Y. Wang, L. Wang, M. Rastegar-Mojarad et al., "Clinical information extraction applications: a literature review," Journal of Biomedical Informatics, vol. 77, pp. 34-49, 2018.

[20] E. Pons, L. M. M. Braun, M. G. M. Hunink, and J. A. Kors, "Natural language processing in radiology: a systematic review," Radiology, vol. 279, no. 2, pp. 329-343, 2016.

[21] M. M. A. Monshi, J. Poon, and V. Chung, "Deep learning in generating radiology reports: a survey," Artificial Intelligence in Medicine, vol. 106, Article ID 101878, 2020.

[22] J. Laserson, C. D. Lantsman, M. Cohen-Sfady et al., "Textray: mining clinical reports to gain a broad understanding of chest $\mathrm{x}$-rays," in International Conference on Medical Image Computing and Computer-Assisted Intervention, pp. 553-561, Springer, Granada, Spain, September 2018.

[23] A. Smit, S. Jain, P. Rajpurkar, A. Pareek, A. Y. Ng, and M. P. Lungren, "Chexbert: combining automatic labelers and expert annotations for accurate radiology report labeling using bert," 2020, https://arxiv.org/abs/2004.09167.

[24] D. A. Wood, J. Lynch, S. Kafiabadi et al., "Automated labelling using an attention model for radiology reports of mri scans (alarm)," 2020, https://arxiv.org/abs/2002.06588.

[25] B. Shin, F. H. Chokshi, T. Lee, and J. D. Choi, "Classification of radiology reports using neural attention models," in Proceedings of the 2017 International Joint Conference on Neural Networks (IJCNN), pp. 4363-4370, IEEE, Anchorage, AK, USA, May 2017.

[26] I. Banerjee, Y. Ling, M. C. Chen et al., "Comparative effectiveness of convolutional neural network (CNN) and recurrent neural network (RNN) architectures for radiology text report classification," Artificial Intelligence in Medicine, vol. 97, pp. 79-88, 2019.

[27] M. Moradi, G. Dorffner, and M. Samwald, "Deep contextualized embeddings for quantifying the informative content in biomedical text summarization," Computer Methods and Programs in Biomedicine, vol. 184, Article ID 105117, 2020.

[28] B. E. Chapman, S. Lee, H. P. Kang, and W. W. Chapman, "Document-level classification of ct pulmonary angiography reports based on an extension of the context algorithm," Journal of Biomedical Informatics, vol. 44, no. 5, pp. 728-737, 2011.

[29] G. Hripcsak, J. H. M. Austin, P. O. Alderson, and C. Friedman, "Use of natural language processing to translate clinical information from a database of 889,921 chest radiographic reports," Radiology, vol. 224, no. 1, pp. 157-163, 2002.

[30] C. Friedman, P. O. Alderson, J. H. M. Austin, J. J. Cimino, and S. B. Johnson, "A general natural-language text processor for clinical radiology," Journal of the American Medical Informatics Association, vol. 1, no. 2, pp. 161-174, 1994.

[31] G. K. Savova, J. J. Masanz, P. V. Ogren et al., "Mayo clinical text analysis and knowledge extraction system (CTAKES): architecture, component evaluation and applications," Journal of the American Medical Informatics Association, vol. 17, no. 5, pp. 507-513, 2010.

[32] E. Scheurwegs, K. Luyckx, L. Luyten, W. Daelemans, and T. Van den Bulcke, "Data integration of structured and unstructured sources for assigning clinical codes to patient stays," Journal of the American Medical Informatics Association, vol. 23, no. e1, pp. e11-e19, 2016.
[33] J. Liu, Z. Zhang, and N. Razavian, "Deep ehr: chronic disease prediction using medical notes," 2018, https://arxiv.org/abs/ 1808.04928.

[34] K. Xu, M. Lam, J. Pang et al., "Multimodal machine learning for automated icd coding," 2018, https://arxiv.org/abs/1810.13348.

[35] M. Jin, M. T. Bahadori, A. Colak et al., "Improving hospital mortality prediction with medical named entities and multimodal learning," 2018, https://arxiv.org/abs/1811.12276.

[36] P. C. G. Simons, A. Algra, M. F. Van de Laak, D. E. Grobbee, and Y. Van der Graaf, "Second manifestations of arterial disease (SMART) study: rationale and design," European Journal of Epidemiology, vol. 15, no. 9, pp. 773-781, 1999.

[37] S. V. Buuren and K. Groothuis-Oudshoorn, "Mice: multivariate imputation by chained equations in $r$," Journal of Statistical Software, vol. 45, pp. 1-68, 2010.

[38] W. Kraaij and R. Pohlmann, "Porter's stemming algorithm for Dutch," in Proceedings of the Informatiewetenschap 1994: Wetenschappelijke bijdragen aan de derde STINFON Conferentie, pp. 167-180, Leiden, Netherlands, June 1994.

[39] M. F. Porter, "Snowball: a language for stemming algorithms," 2001, http://snowball.tartarus.org/.

[40] D. Blei, A. Ng, and M. Jordan, "Latent dirichlet allocation," Journal of Machine Learning Research, vol. 3, no. 1, pp. 993-1022, 2003.

[41] T. Mikolov, I. Sutskever, K. Chen, G. S. Corrado, and J. Dean, "Distributed representations of words and phrases and their compositionality," in Advances in Neural Information Processing Systems, pp. 3111-3119, MIT press, Cambridge, UK, 2013.

[42] T. Mikolov, K. Chen, G. Corrado, and J. Dean, "Efficient estimation of word representations in vector space," 2013, https://arxiv.org/abs/1301.3781.

[43] S. Tong and D. Koller, "Support vector machine active learning with applications to text classification," Journal of Machine Learning Research, vol. 2, pp. 45-66, 2001.

[44] M. Schuster and K. K. Paliwal, "Bidirectional recurrent neural networks," IEEE Transactions on Signal Processing, vol. 45, no. 11, pp. 2673-2681, 1997.

[45] N. Srivastava, G. Hinton, A. Krizhevsky, I. Sutskever, and R. Salakhutdinov, "Dropout: a simple way to prevent neural networks from overfitting," The Journal of Machine Learning Research, vol. 15, no. 1, pp. 1929-1958, 2014.

[46] P. Branco, L. Torgo, and R. Ribeiro, "A survey of predictive modelling under imbalanced distributions," 2015, https:// arxiv.org/abs/1505.01658.

[47] W. Van Amsterdam, J. Verhoeff, P. de Jong, T. Leiner, and M. Eijkemans, "Eliminating biasing signals in lung cancer images for prognosis predictions with deep learning," NPJ Digital Medicine, vol. 2, no. 1, pp. 1-6, 2019.

[48] Y.-W. Lin, Y. Zhou, F. Faghri, M. J. Shaw, and R. H. Campbell, "Analysis and prediction of unplanned intensive care unit readmission using recurrent neural networks with long short-term memory," PLoS One, vol. 14, no. 7, Article ID e0218942, 2019.

[49] A. Sammani, A. Bagheri, P. .G. M. Van Der Heijden et al., "Automatic multilabel detection of icd10 codes in Dutch cardiology discharge letters using neural networks," NPJ Digital Medicine, vol. 4, no. 1, pp. 37-10, 2021.

[50] W. Chen, Z. Lu, L. You, L. Zhou, J. Xu, and K. Chen, “Artificial intelligence-based multimodal risk assessment model for surgical site infection (amrams): development and validation study," JMIR Medical Informatics, vol. 8, no. 6, Article ID e18186, 2020.

[51] H. Suresh, N. Hunt, A. Johnson, L. A. Celi, P. Szolovits, and M. Ghassemi, "Clinical intervention prediction and understanding using deep networks," 2017, https://arxiv.org/abs/ 1705.08498 . 